\title{
Prevalence of Variant Anatomy of Paranasal Sinuses in Computed Tomography and its Correlation to Sinusitis:
}

\author{
Devimeenal Jagannathan ${ }^{1}$, Gopinathan Kathirvelu ${ }^{2}$, Fouzal Hithaya ${ }^{3}$ \\ ${ }^{\text {I}}$ (Professor, Department Of Radio-Diagnosis, Government Kilpauk Medical College - Chennai - India) \\ ${ }_{2}^{2}$ (Associate Professor, Department Of Radio-Diagnosis, Government Kilpauk Medical College - Chennai- \\ India) \\ ${ }_{3}^{3}$ (Resident, Department Of Radio-Diagnosis, Government Kilpauk Medical College - Chennai-India)
}

\begin{abstract}
This study aims at identifying the prevalence of multiple variants in CT of paranasal sinuses(PNS) done in our institution and to find out correlation to sinusitis if any. A retrospective analysis of 200 cases (100 in sinusitis group and 100 in asymptomatic group) of CT PNS was done at our institution. The prevalence of variants including those with surgical importance were studied. Comparison was done using Pearson's Chi square test.The most common variant was deviated nasal septum, followed by lamellar concha followed by agger nasi cell in both the groups. Though variants were found in higher numbers in sinusitis group, there was no statistical significance in their prevalence between both the groups. It is customary to find innumerable variant anatomy in CT of paranasal sinuses. But there is no scientific correlation for the variants as causative factors of sinusitis. However the variant anatomy retains its significance to be mentioned in reporting in the surgical perspective.
\end{abstract}

Keywords: CT PNS, anatomical variants, sinusitis, minimally invasive surgeries

\section{Introduction}

Sinusitis is one of the most common and important presentations in patients presenting to ENT (ear, nose, throat) outpatient department. Although sinusitis is diagnosed clinically, CT PNS (Computed tomography paranasal sinuses) has become the easily accessible and feasible investigation in the specific diagnosis of sinusitis. The paranasal sinus anatomy is complex and is subject to innumerable and inconsistent variations. CT PNS remains the investigation of choice in evaluation of paranasal sinus anatomy, its pathology and the variations. The correlation between the presence of anatomical variants to sinusitis is highly debatable. Many studies have been conducted earlier in this regard. Though there are studies that prove a positive correlation between the anatomical variants to sinusitis there are equal number of studies which say that there exists no such correlation. The anatomical variants disturbing the osteomeatal complex function (common channel formed linking frontal, maxillary, anterior and posterior ethmoidal sinuses to the middle meatus) is supposed to increase the risk of sinusitis. Some of the osteomeatal anatomical variants that may predispose to meatal narrowing and even obstruction include DNS (deviated nasal septum), concha bullosa, paradoxically bent middle turbinate, agger nasi cells and Haller cells. CT PNS has also an invaluable role in presurgical evaluation of minimally invasive FESS (Functional endoscopic sinus surgery), aimed at rectification of osteomeatal complex pathology. The uncinate anatomy, depth of olfactory fossa, anterior clinoid pneumatisation, intersphenoid septum deflection are some of the most important anatomical delicacies that have to be predefined prior to FESS to avoid devastating injuries. This study aims to find out the types of anatomical variants in CT PNS, their prevalence, correlation to sinusitis and significance of the same prior to minimally invasive surgeries.

\subsection{Inclusion criteria}

\section{Materials And Methods}

This retrospective study was conducted in Government Kilpauk Medical College, Department of Radio diagnosis by analyzing the patients who underwent CT PNS from September 2016 - February 2017 for a period of six months. 100 patients with clinical and radiological features of sinusitis referred from department of ENT for CT PNS were included in the study group. Clinical features like nasal obstruction, rhinorrhoea, facial pain were considered as positive clinical findings for the study group. Mucosal thickening in the sinuses $>1 \mathrm{~mm}$ were considered as positive radiological findings. 100 patients without any or $<1 \mathrm{~mm}$ mucosal thickening in the sinuses were considered in the control group. The study was done after obtaining ethical committee approval and informed consent from the patients.

\subsection{Exclusion criteria}

Patients with fungal sinusitis, sinonasal polyposis, sinonasal malignancies, trauma were excluded. 


\subsection{CT protocol}

The study was done using a MDCT Scanner TOSHIBA, using120 Kvp, $100 \mathrm{mAs}$, 220mm FOV and slice thickness of $4 \mathrm{~mm}$. Axial imaging was done with $4 \mathrm{~mm}$ thickness parallel along the orbito meatal plane extending inferiorly from upper dental arch to roof of frontal sinuses superiorly ${ }^{(1)}$. Coronal images were obtained with $4 \mathrm{~mm}$ thickness, in a plane perpendicular to hard palate, from anterior wall of frontal sinus to posterior wall sphenoid sinus.

\section{Results}

The study population ranged from $12-76$ years. $98(49 \%)$ were males and $102(51 \%)$ were females. Paranasal variants were present in $89 \%$ of the cases. The classification of variants is shown in Fig1. The distribution of the anatomical variants is shown in Table 1. Surgically important variants are tabulated in Table 2. The most common variant identified in our study was deviated nasal septum (56.5\%). The second most common variant was lamellar concha (39\%) followed by agger nasi (37\%). The results were analysed using SPSS statistics software 23.0 version. The statistical test used was Pearson Chi square test. The probability value of 0.05 was considered significant level. The results are tabulated in Table 3. There was no statistical difference in the prevalence of the anatomical variants between the two groups studied. The representative coronal CT PNS images are shown in figures 2 to 8 .

\section{Discussion}

Paranasal variants were found in $99.8 \%$ of the patients ${ }^{(2)}$ in a study conducted by Dasar U et al which was $89 \%$ in our study.The nasal septum is formed by septal cartilage, perpendicular plate of ethmoid and horizontal plate of vomer ${ }^{(2)}$. Malalignment of the three components results in deviation to one side or other with or without a septal spur ${ }^{(2)}$. Approximately spur is present in $1 / 3^{\text {rd }}$ of patients with deviated septum. We considered deviation as any asymmetric curvature of septum. We reported a prevalence of $56.5 \%$ in this study. The prevalence of deviated nasal septum in literature ranges from $19.4 \%-74 \%{ }^{(3)}$. Perez et al reported it to be $80 \%$. It was as low as $19.4 \%$ according to Smith et $\mathrm{al}^{(4)}$ as they followed the strict criteria of deviation $>4 \mathrm{~mm}$. DNS causing a reduction in osteomeatal unit's critical area, impairing mucociliary activity and drainage is supposed to predispose to sinusitis. However there was no significant correlation to sinusitis in the study done by Shpilberg et al

Agger nasi are the anterior most ethmoidal cells situated inferolateral to frontal recess. Their reported prevalence is highly variable from $3-100 \%$. The size of the cell may compensate the frontal recess and also the anterior part of middle meatus ${ }^{(5)}$. Kaygusuz et al ${ }^{(6)}$ reported a prevalence of $61 \%$ in their study but without a significant etiology in frontal sinusitis. In our study the prevalence was $37 \%$. The prevalence of ethmoidal bulla is $30 \%$ which can cause sinusitis by obstructing the meatus. Tiwari et al ${ }^{(7)}$ in their study reported large ethmoidal bulla in $65 \%$ of patients with chronic sinusitis. We found a prevalence of $18.5 \%$ in our study. Haller cells are the ethmoidal cells that grow into the orbital floor that may narrow the maxillary ostium predisposing to sinusitis. The reported prevalence range is $10-62 \%{ }^{(3)}$. In our study it was $18.5 \%$.

Concha bullosa ${ }^{(8)}$ is pneumatisation of bulbous portion of the middle turbinate. Prevalence varies from $14-67.5 \%{ }^{(3)}$. Stalman et al ${ }^{(9)}$ defined concha bullosa as when atleast $50 \%$ of vertical height of middle turbinate was pneumatised. It is supposed to interfere with mucociliary clearance in and around middle meatus. Pneumatisation of the vertical lamella of the concha is called the lamellar concha. In our study the prevalence was 39\% which is consistent with reported valence of $9.6-46 \%{ }^{(3)}$. Reverse curve of middle turbinate with convexity laterally results in a paradoxical middle turbinate ${ }^{(10)}$ that can cause sinusitis by impingement of the middle meatus. In our study the prevalence was $20.5 \%$.

Horizontal uncinate, hypertrophied uncinate, uncinate bullae are the uncinate variants with reported prevalence of 5\% each. These can narrow the hiatus semilunaris or ethmoidal infundibulum. Uncinate variants were found in $9 \%$ of the cases.

Michael et $\mathrm{al}^{(11)}$ proposed a score for standardization of variants of pneumatisation using 18 items called Assessment of pneumatisation of paranasal sinuses score (APSS). In our study the pneumatisation identified were that of superior turbinate, crista galli, anterior clinoid process, lateral recess, hard palate , pterygoid plates, and nasal septum. Superior turbinate was pneumatised in $4.5 \%$ of cases which is less compared to the reported incidence of $27 \%{ }^{(3)}$. There was no case of pneumatised inferior turbinate in our study and previous studies also reported it as a very rare variant ${ }^{(3)}$. Crista galli pneumatisation was present in $22 \%$ of our cases. Som et al ${ }^{(12)}$ in their study compared crista galli pneumatisation in three groups divided as > 7years, $7-12$ years, $<12$ years. They concluded that though crista galli is part of ethmoid bone, pneumatisation of it extends from the frontal sinus and its pathology might direct the clinician to frontal sinus as a primary source. Pneumatisation of hard palate extends the floor of maxillary sinus inferior to the nasal cavity floor seen in $7 \%$ of the cases. Frontal sinus pneumatisation may vary to great extent. Frontal sinus may be aplastic, pneumatisation 
may be below or above the supra orbital rim in the superior extent. Laterally it may or may not extend lateral to medial edge of orbit or may extend lateral to mid pupillary line. Frontal recess ${ }^{(13)}$ is bounded medially by middle turbinate and laterally by lamina papyracea. The anterior ethmoidal air cells that are pneumatised in this area are the frontal recess cells. The reported incidence is $20-40 \%$. In our study frontal recess cells were present in $12 \%$ of the cases. Kuhn classified it into 4 types, type 1: single frontal cell above agger nasi, type 2 : tier of cells in frontal recess, type 3: large ethmoidal air cell type 4: totally isolated cell within the frontal sinus.

Onodi cell, the posterior ethmoidal air cell was first reported by Adolf Onodi in 1904. The reported incidence is $8-24 \%$. In our study we found onodi cells in $10 \%{ }^{(14)}$. It lies superior to sphenoid sinus. Its close proximity to optic nerve, internal carotid artery makes its diagnosis imperative prior to FESS. Variants of optic nerve in relation to sphenoidal sinus ${ }^{(15)}$ are: type 1: optic nerve is adjacent to sphenoid sinus without indentation, type 2: nerve is adjacent to sphenoid sinus indenting the wall, type 3: nerve courses through sphenoid sinus and type 4: nerve is adjacent to sphenoid sinus and posterior ethmoidal cell. In our study the distribution into 4 types were $81 \%, 9 \%, 8 \%, 2 \%$ respectively. Anatomic configurations that predispose optic nerve to injury are type 2, 3, anterior clinoid process pneumatisation, bony dehiscence. Anterior clinoid pneumatisation was frequently associated with type $2(31 \%)$, type 3(54\%). Lateral recess or pterygoid recess pneumatisation ${ }^{(16)}$ is defined as pneumatisation extending beyond the imaginary line joining the foramen rotundum and Vidian's nerve. This sets the neurovascular structures prone for injury during endonasal approach. In our study its prevalence was $14 \%$.

The sphenoid sinus is an important route of surgery used by neurosurgeons in skull base surgeries with vital structures like cavernous sinus, carotid artery and optic nerves around it. The pneumatisation is classified into three types presellar, sellar and conchal. Presellar type has moderate air cavity without bulge of the sellar floor into the sphenoid sinus, in sellar type pneumatisation extends beyond the tuberculum sella into body of sphenoid as far as the clivus. In conchal type sphenoid sinus is a bony obstacle without air cavity that obliterates the direct access to the sella. The reported prevalence is $83 \%, 16.5 \%, 0.5 \%{ }^{(17)}$. However the extent of pneumatisation of sphenoid sinus and its relation to sella turcica is best evaluated by MRI.

The height of lateral cribriform plate lamella ${ }^{(18)}$ determines the depth of the olfactory fossa, the base is formed by fovea ethmoidalis which separates anterior ethmoidal cells from anterior cranial fossa. Kero established a classification criteria in 1962. Type 1: 1-3mm (26.3\%), type 2: 4-7 mm(73.3\%), type 3: 8 - 16mm $(0.5 \%)$. The increased depth of olfactory fossa paves way for an increased chance of an accidental injury to anterior cranial fossa not only during FESS but also in trauma and also in setting of tumour erosion. Limitations Since we studied the axial and coronal images the pattern of sellar pneumatisation could not be included in this study which is best studied in sagittal image.

\section{Figures and Tables}

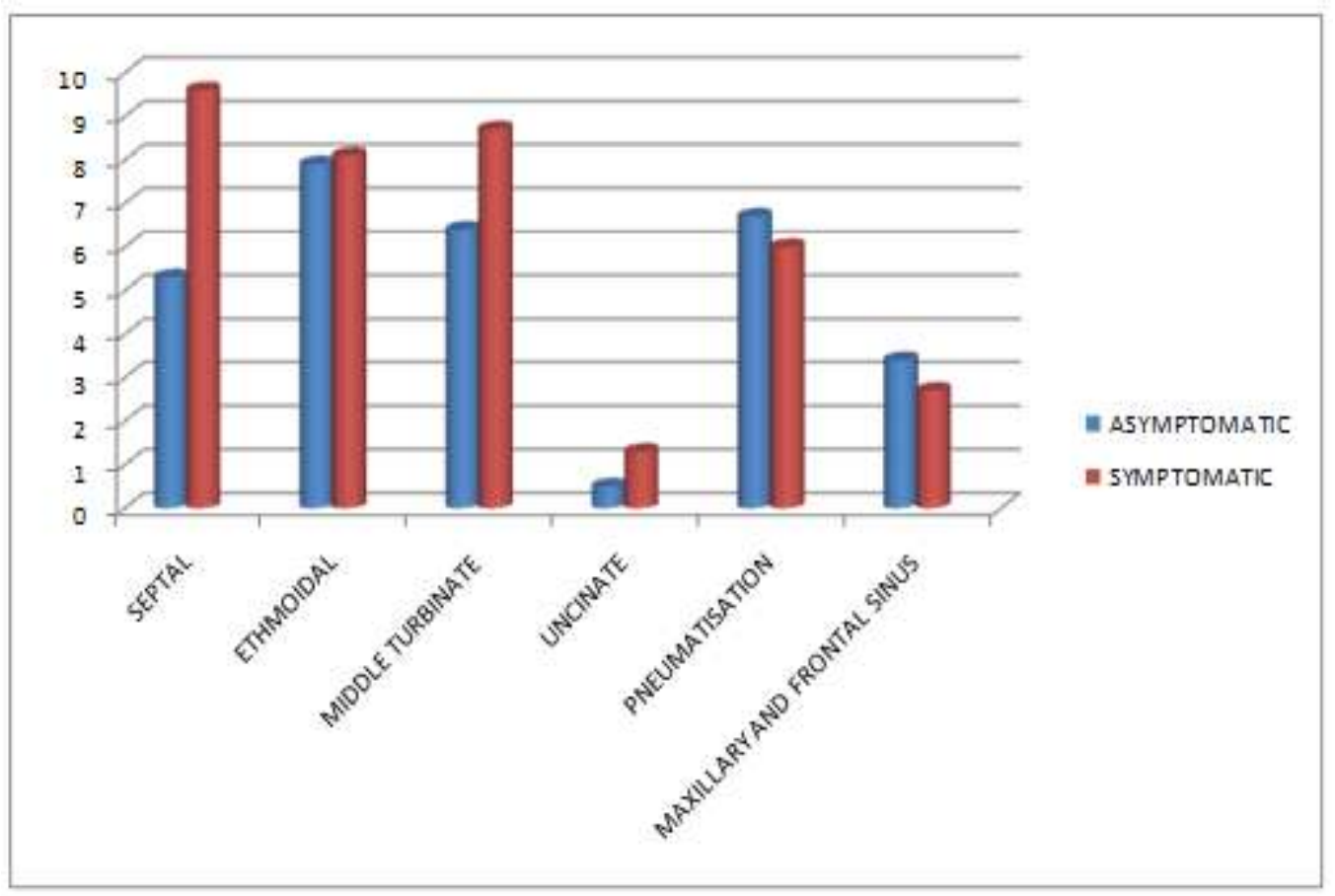

Fig 1 Distribution of anatomical variants in asymptomatic and symptomatic groups 


\begin{tabular}{|c|c|c|}
\hline Table 1 & PNS Variants & \\
\hline Variants & Asymptomatic & Symptomatic \\
\hline \multicolumn{3}{|l|}{ Septal variants: } \\
\hline DNS & 43 & 70 \\
\hline Spur & 10 & 26 \\
\hline \multicolumn{3}{|l|}{ Ethmoidal variants: } \\
\hline Agger nasi & 36 & 38 \\
\hline Ethmoidal bulla & 18 & 19 \\
\hline Haller cell & 14 & 15 \\
\hline Onodi cell & 11 & 9 \\
\hline \multicolumn{3}{|l|}{ Middle turbinate variants: } \\
\hline Lamellar concha & 40 & 38 \\
\hline Concha bullosa & 13 & 28 \\
\hline Paradoxical middle turbinate & 11 & 21 \\
\hline \multicolumn{3}{|l|}{ Uncinate variants: } \\
\hline Horizontal & 3 & 7 \\
\hline Pnuematised & 2 & 4 \\
\hline Hypertrophied & 0 & 2 \\
\hline \multicolumn{3}{|l|}{ Pneumatisation variants: } \\
\hline Superior turbinate & 4 & 5 \\
\hline Crista galli & 23 & 21 \\
\hline Anterior clinoid process & 20 & 18 \\
\hline Lateral recess of sphenoid & 15 & 13 \\
\hline Hard palate & 5 & 2 \\
\hline Nasal septum & 0 & 1 \\
\hline \multicolumn{3}{|l|}{ Maxillary sinus variants: } \\
\hline Septum & 3 & 5 \\
\hline Accessory ostium & 5 & 6 \\
\hline \multicolumn{3}{|l|}{ Frontal sinus variants: } \\
\hline Frontal recess cells & 13 & 10 \\
\hline Extensive pneumatisation & 13 & 6 \\
\hline
\end{tabular}

Table 2 Surgically important variants

\begin{tabular}{|l|l|l|}
\hline Dehiscent optic nerve tubercle types: & Asymptomatic & Symptomatic \\
\hline I & 80 & 82 \\
\hline II & 11 & 7 \\
\hline III & 8 & 8 \\
\hline IV & 1 & 3 \\
\hline LCPL (Lateral cribriform plate lamella) types: & & \\
\hline I & 27 & 24 \\
\hline II & 72 & 75 \\
\hline III & 1 & 1 \\
\hline
\end{tabular}

Table 3 Statistical significance of anatomical variants between the asymptomatic (control group) and symptomatic (study group)

\begin{tabular}{|l|l|l|}
\hline S.no & Anatomical variants & p value \\
\hline 1. & Septal variants & 0.262 \\
\hline 2. & Ethmoidal variants & 0.962 \\
\hline 3. & Middle turbinate variants & 0.071 \\
\hline 4. & Uncinate variants & 0.642 \\
\hline 5. & Pneumatisation variants & 0.798 \\
\hline 6. & Maxillary variants & 0.729 \\
\hline 7. & Frontal sinus variants & 0.429 \\
\hline
\end{tabular}

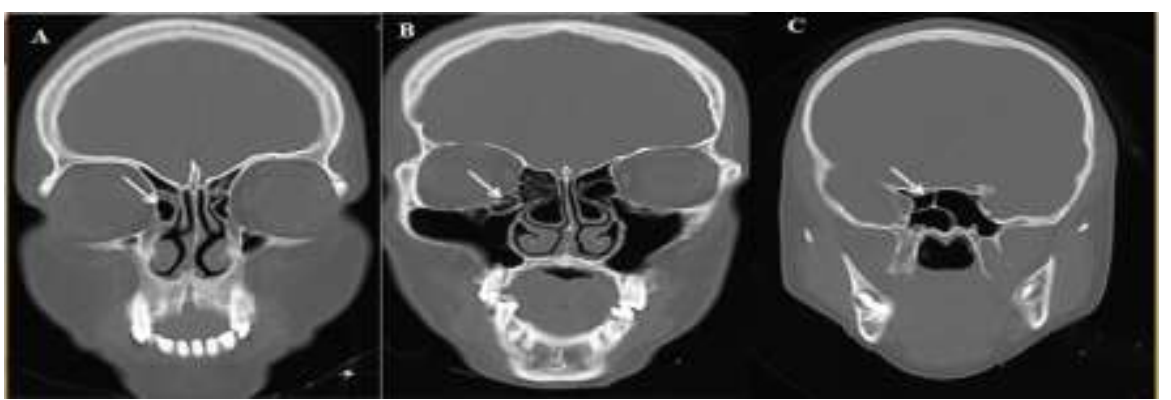

Figure 2 A - Agger nasi (white arrow), B - Haller cell (white arrow), C - Onodi cell (white arrow) 


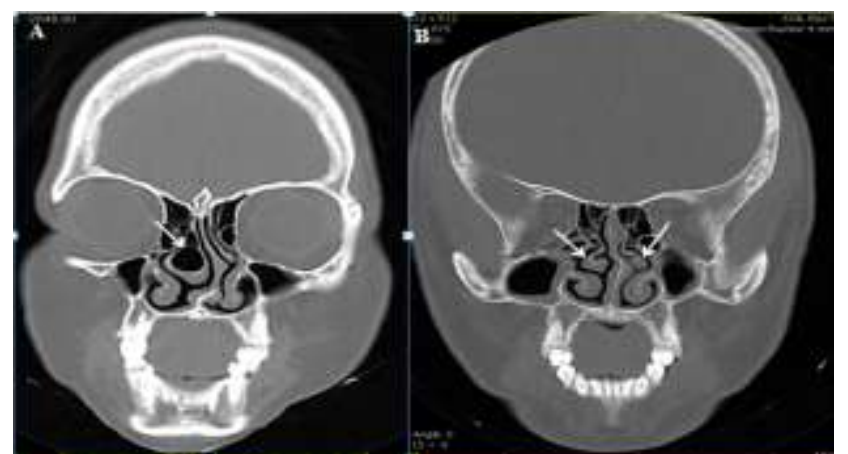

Figure 3 A - Deviated nasal septum with right concha bullosa (white arrow), B - paradoxical middle turbinates (white arrows)

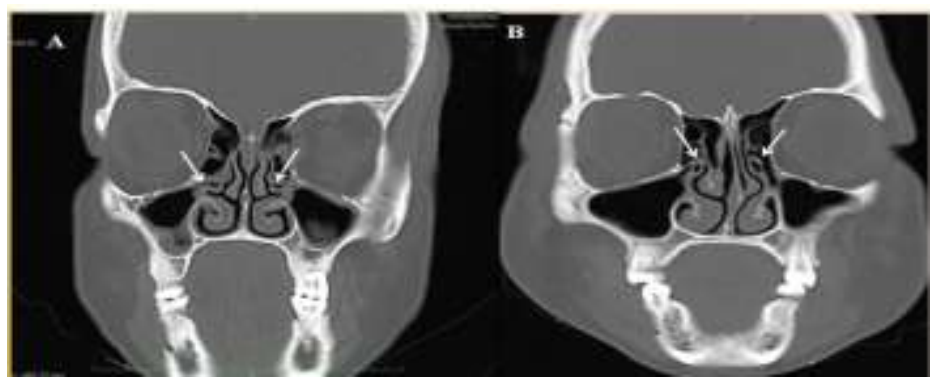

Figure 4 A- Horizontal uncinate process (white arrows), B - pneumatised uncinate process (white arrows)

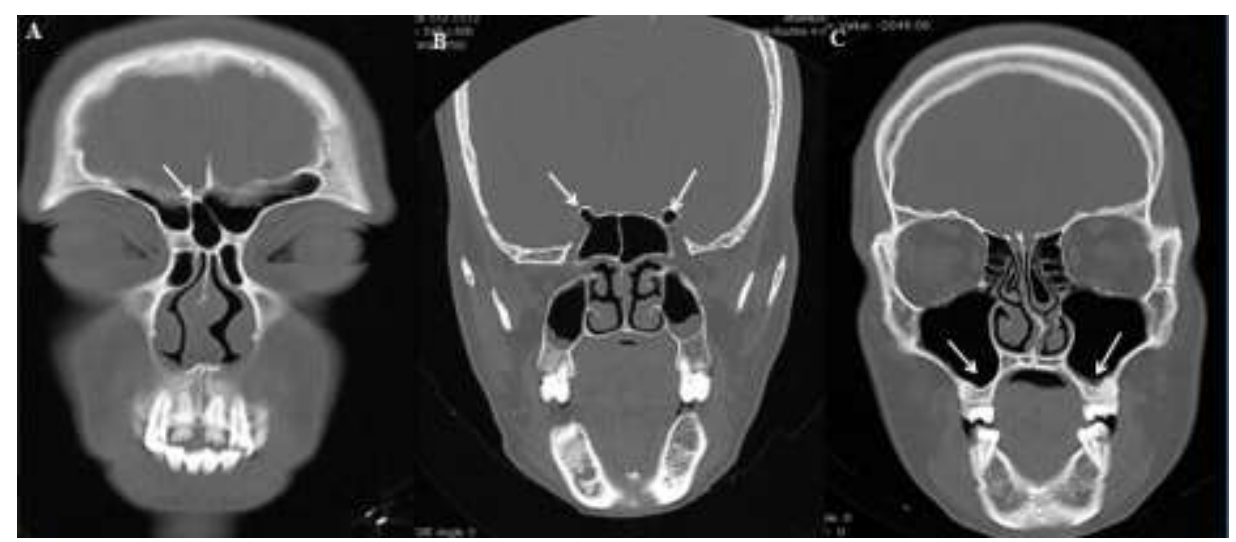

Figure 5 Pneumatisation variants - A - Crista galli (white arrow), B - Anterior clinoid process (white arrow), C - Hard palate (white arrow)

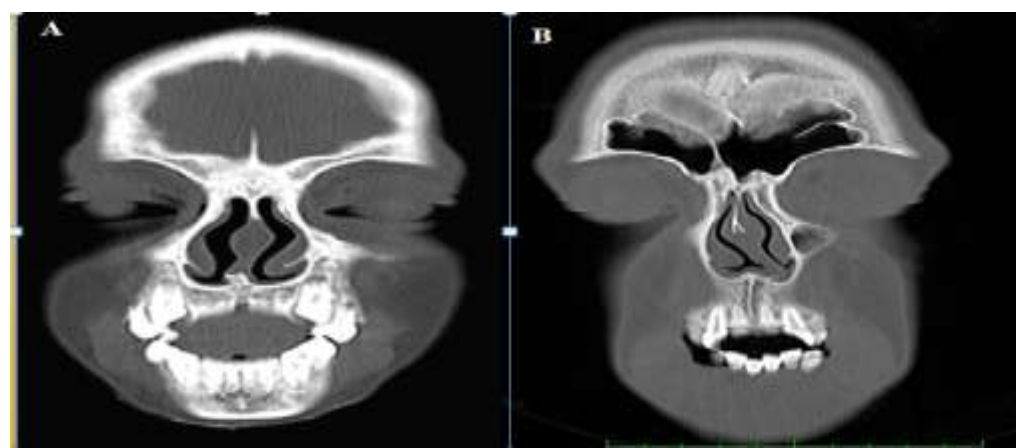

Figure 6 Frontal sinus variants $\mathbf{A}$ - Aplastic , B - pneumatisation extending beyond medial edge of orbit to mid pupillary line 


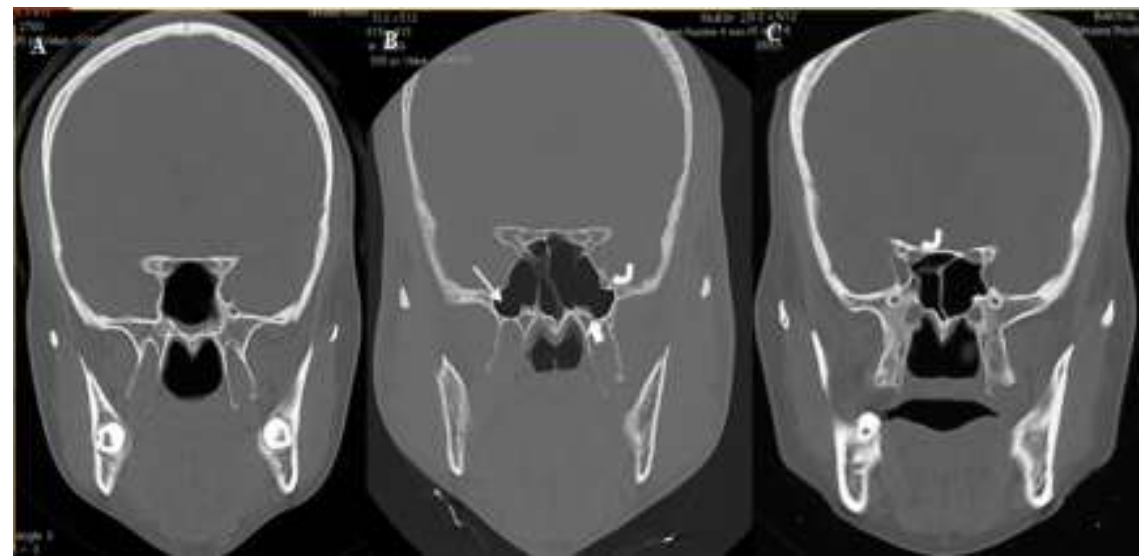

Figure 7 Sphenoid sinus variants $\mathbf{A}$ - absence of intersphenoid septum, $\mathbf{B}$ - lateral recess (white arrow) exposing foramen rotundum ( thick curved arrow) and Vidian's nerve (thick arrow), $\mathbf{C}$ - Dehiscent optic nerve (thick curved arrow)

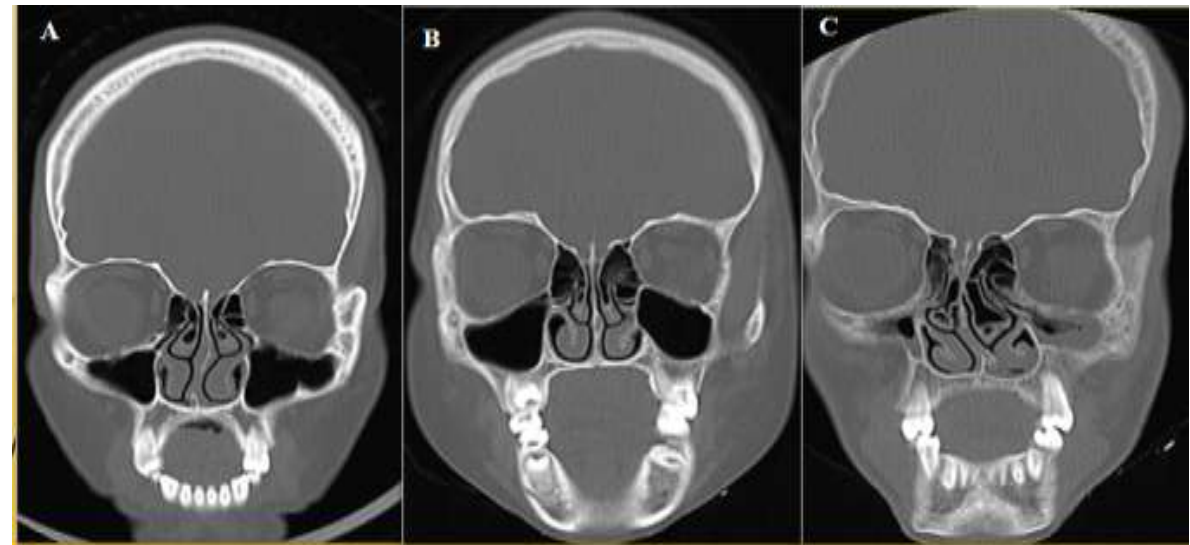

Figure 8 Lateral cribriform plate lamella - Kero's classification A - Type I, B - Type II, C - Type III

\section{Conclusion}

The fact that variant anatomy predisposes to sinusitis is controversial with many previous equivocal studies. In our study we conclude that the presence of variants is not a causative factor for sinusitis but the same gains importance in surgical perspective.

\section{References}

[1]. Perez - PINAS, I., et al. "Anatomical variations in the human paranasal sinus region studied by CT." Journal of anatomy 197.2 (2000): 221-227.

[2]. Dasar, Ufuk, and Erkan Gokce. "Evaluation of variations in sinonasal region with computed tomography." World journal of radiology 8.1 (2016): 98 .

[3]. Shpilberg, Katya A., et al. "CT of anatomic variants of the paranasal sinuses and nasal cavity: poor correlation with radiologically significant rhinosinusitis but importance in surgical planning." American Journal of Roentgenology 204.6 (2015): 1255-1260.

[4]. Smith, Kyle D., et al. "The prevalence of concha bullosa and nasal septal deviation and their relationship to maxillary sinusitis by volumetric tomography." International journal of dentistry 2010 (2010).

[5]. Reddy, Uma Devi Murali Appavoo, and Bhawna Dev. "Pictorial essay: Anatomical variations of paranasal sinuses on multidetector computed tomography-How does it help FESS surgeons?" The Indian journal of radiology \& imaging 22.4 (2012): 317.

[6]. Kaygusuz, Ahmet, et al. "Sinonasal anatomical variations: their relationship with chronic rhinosinusitis and effect on the severity of disease - a computerized tomography assisted anatomical and clinical study." Indian Journal of Otolaryngology and Head \& Neck Surgery 66.3 (2014): 260-266.

[7]. Tiwari, Rashi, and Rashmi Goyal. "Study of anatomical variations on CT in chronic sinusitis." Indian Journal of Otolaryngology and Head \& Neck Surgery 67.1 (2015): 18-20.

[8]. Aramani, Anita, R. N. Karadi, and Saurabh Kumar. "A Study of Anatomical Variations of Osteomeatal Complex in Chronic Rhinosinusitis Patients-CT Findings." Journal of clinical and diagnostic research: JCDR 8.10 (2014): KC01.

[9]. Stallman, Jamie S., Joao N. Lobo, and Peter M. Som. "The incidence of concha bullosa and its relationship to nasal septal deviation and paranasal sinus disease." American Journal of neuroradiology 25.9 (2004): 1613-1618.

[10]. Azila, A., et al. "The prevalence of anatomical variations in osteomeatal unit in patients with chronic rhinosinusitis." The Medical journal of Malaysia 66.3 (2011): 191-194

[11]. Marino, Michael J., et al. "Assessment of pneumatization of the paranasal sinuses: a comprehensive and validated metric." International forum of allergy \& rhinology. 2015 
[12]. Som, P. M., et al. "Crista galli pneumatization is an extension of the adjacent frontal sinuses." American Journal of Neuroradiology 30.1 (2009): 31-33.

[13]. Eweiss, Ahmed Z., and Hisham S. Khalil. "The prevalence of frontal cells and their relation to frontal sinusitis: a radiological study of the frontal recess area." ISRN otolaryngology 2013 (2013).

[14]. Cherla, Deepa V., et al. "The central Onodi cell: A previously unreported anatomic variation." Allergy \& Rhinology 4.1 (2013):

[15]. DeLano, Mark C., F. Y. Fun, and S. James Zinreich. "Relationship of the optic nerve to the posterior paranasal sinuses: a CT anatomic study." American journal of neuroradiology 17 (1996): 669-675.

[16]. Mohebbi, Alireza, et al. "The sphenoid sinus, foramen rotundum and vidian canal: a radiological study of anatomical relationships." Brazilian journal of otorhinolaryngology (2016).

[17]. Sevinc, Ozdemir, et al. "Anatomic Variations of Sphenoid Sinus Pneumatization in a Sample of Turkish Population: MRI Study." Int. j. morphol 32.4 (2014): 1140-1143.

[18]. Bista, M., et al. "Computed tomographic assessment of lateral lamella of cribriform plate and comparison of depth of olfactory fossa." Journal of the Nepal Medical Association 49.178 (2010).

[19]. M Ozaki, Y. Adachi, Y. Iwahori, and N. Ishii, Application of fuzzy theory to writer recognition of Chinese characters, International Journal of Modelling and Simulation, 18(2), 1998, 112-116. 